\title{
The Behaviour of Gas Bubble during Rest Period of Pulse-Activated Electrolysis Hydrogen Production
}

\author{
Thanet Vilasmongkolchai, Roongrojana Songprakorp, Kaokanya Sudaprasert \\ Division of Energy Technology, School of Energy, Environment and Materials, King Mongkut's University of Technology Thonburi, Bangkok, \\ Thailand
}

\begin{abstract}
The pulse-activated electrolyzer has been developed and used for several years. With the capability of enhancing the efficiency of an electrolytic process and easy operation, this technique becomes an interesting process for hydrogen production. Unfortunately during electrolytic reaction, the creation of bubbles becomes a reaction inhibitor and consumes energy. This paper aims to study the proper rest period that gives the bubble free rise-off the solution without additional bubble created. The mathematical method and acoustic emission method were used for investigation of bubble's rising velocity. The result shows that the variation of rest period on pulse-activated makes production efficiency enhanced. For the practicality of use and set control parameters, duty cycle and frequency were demonstrated instead of rest period.
\end{abstract}

\section{Introduction}

Due to energy crisis and global warming awareness, the study of renewable energy also up-to-date challenged research problem. Hydrogen is one of alternative clean energy with a possibly use in several (e.g. direct combustion, fuel cell). Nowadays, the most economical hydrogen production process needs to use fossil fuels. The steam reforming process is not only consuming hydrocarbon products, but also releasing carbon monoxide. The electrolytic process is the use of electrical field activated with electrodes so, the water split into hydrogen and oxygen. This method is an advantage of producing extremely pure hydrogen and oxygen without any carbon emission. The pulse-activated has been implemented to use with electrolysis process for two decades with the good result in productivity and efficiency enhancement. According to the previous study, the electrical circuit has been used to describe electrolysis system [1], [2] and the results were revealed that the natural frequency make system's efficiency improved [2], [3].

Unlike this study, the research was focused on the increasing of Ohmic's loss due to the bubble created. Based on the main idea that has been proved in several literatures, during the electrolytic hydrogen generation process, gaseous products make the resistivity of the system increases and hence lower system efficiency [1], [4]-[6], so the degasing is the effective method to achieve higher efficiency. The rest period of the pulse-activated is the advantage to allow the bubbles rising-off the solution with no more bubble created and make system neutralized before getting activated in next cycle. It seems nonsense when getting longer rest period could reduce loss and less productivity, then the shortest rest period should be where there is no bubble in electrolyte.

\section{Approach and experiment procedure}

When current supply to the electrolysis cell, reaction occurred and gas bubble created. The bubble form on electrode surface then detach and rise-off electrolyte surface. Before the bubble rise-off the solution, this stage caused more energy consuming and augment higher resistivity. In addition, the bubble coverage reduce active area of electrode and make reaction prohibited. These unavoidable problems could basically solve by making bubble rise-off the solution quicken to get higher efficiency. So, the parameters that effect bubble behavior (e.g. bubble size, bubble rising velocity) should be considered.

This research uses a combination method to study a role of a rest period in hydrogen electrolysis efficiency improvement. Fig. 1 shows an overall structure of the system, the arrows connecting the block diagrams demonstrate the electrical flow (solid line) and signal flow (dashed line). The electrolyzer used $20 \% \mathrm{wt} \mathrm{KOH}$ solution as electrolyte under atmospheric pressure and the temperature was controlled at $353 \mathrm{~K}$. Both electrodes were made of stainless steel connected to the adjustable current source power supply of $10-50 \mathrm{~A}$ in range which used to control activation parameters via a pulse generator. The gases obtained from the cathode and the anode were collected separately in gas tubes. The volume of gas produced was measured using gas mass flow meter, and re-checked with acoustic emission technique. 
Therefore, broadband acoustic emission sensors were installed beside both electrodes in the chamber.

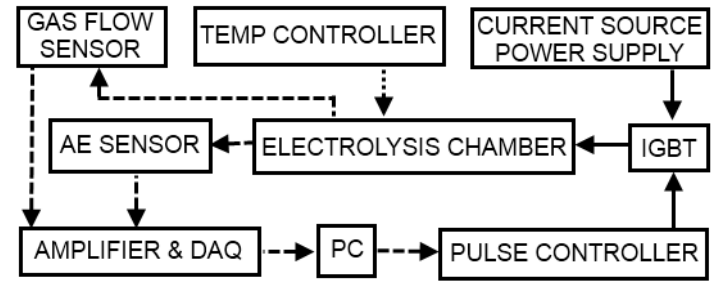

Figure 1. Overall structure of the system.

\subsection{Bubble}

The gaseous product of electrolyzer is generally known as a reaction inhibitor [4]-[6]. The coverage bubble on electrodes is directly affected with electrolytic resistivity, so the rest time in pulse-activated achieved the system's parameter reset, with no current input and the existing bubbles rise off the electrolyte solution. From the simple law of motion, the rest period $\left(t_{r s t}\right)$ can be calculated by

$$
t_{r s t}=y / v_{t}
$$

According to equation (1), the average distance of bubbles traveling in $\mathrm{y}$-axis $(y)$ has been assumed as no effect in bubble diffusion. The bubble's terminal velocity $\left(v_{t}\right)$ lies on the bubble size. The bigger can reach the top surface quicker although drag force would be greater. It is assumed that all bubbles are spherical (with constant shape), inviscid and irrotational flow outside bubbles, no boundary layer separation, and no internal flow circulation inside bubbles. From the previous study [7], the rise velocity of the single bubble $\left(v_{b}\right)$ was calculated and the free stream velocity or swarm velocity $\left(v_{s}\right)$ has been derived as a function of the voidage $(\varepsilon)$. Hence, the mathematical estimation of rest period indicated as equation (2) with the following parameters: bubble's radius $(r)$, difference of density between inside and outside bubble $(\Delta \rho)$, gravitational acceleration $(g)$, electrolyte's dynamic viscosity $(\mu)$.

$$
t_{r s t}=9 y \cdot \mu /\left(r^{2} \cdot \Delta \rho \cdot g\right)(1-\varepsilon)^{3}
$$

So, the minimum rest period mainly depends on the bubble size and the void fraction. In other words, the activation current was a significant parameter because it has a direct effect on the void fraction. Although the void fraction usually become a lower value due to rest period, but the greater activation current always make greater value of void fraction.

\subsection{Pulse activation}

The past literature on the pulse-activated hydrogen electrolysis presented the advantage of activation frequency that led more efficiency [8]. So, the later researches explained the frequency response behavior of the pulse-activated to the influence of natural frequency using the electric circuit transfer model and set the activation frequency in range of kilohertz and over [2], [3]. According to earlier experiments, all results showed the efficiency enhancement on the natural frequency activation. However, the frequency selected in modeling should be migrated during activation because of eigenvalue migration effect, and the experimental parameter and environment were sensitive. In practice, the activation frequency cannot be estimated as a constant. Following this idea to measurement and tracking natural frequency, the control unit and sensor with high precision and high sensitivity are needed. Consequently, it was very difficult to maintenance the system depending on the sophistication of the system without high cost. Moreover, the measurement of some parameters cannot be taken accurately during activation. Hence, the low frequency should be used in this research to study the effect of bubble to rest period of a pulse activated without natural frequency awareness.

\subsection{Bubble observation}

At present, the study of bubble's dynamic using high speed camera is popular, with the advantage in a good accuracy estimating bubble's size in a high volume of bubble and/or in depth dimension.

Acoustic Emission (AE) is one of the non-destructive testing methods that uses the phenomenon of instantaneous acoustic waves propagating in media when a physical change occurs in the media's structure. So, in electrochemical reaction, AE has been used widespread for electrode monitoring and corrosion [9]-[11]. According to the experiments, natural frequency of the breaking hydrogen bubble is about $120-150 \mathrm{kHz}$, and the parameter "AE count" is varied depending on gas production rate.

The measurement of bubble breaking's natural frequency is an indirect measurement of bubble size. The determination of the breaking acoustic frequency $\left(f_{b}\right)$ corresponding to the bubble radius $\left(r_{0}\right)$ can be formulated as equation (3) [11] with the following parameters; static pressure in the fluid $\left(P_{0}\right)$, specific heats for the gas $(\gamma)$, Newtonian fluid of constant density $\left(\rho_{0}\right)$.

$$
f_{b} \approx \operatorname{sqrt}\left(\left(3 \gamma \cdot P_{0}\right) / \rho_{0}\right) /\left(2 \pi \cdot r_{0}\right)
$$

\section{Results and discussion}

\subsection{Bubble and rising velocity}

In the experimental setup, the measured parameters include activation peak current, averaged activation current, voltage of electrolyzer, gas production rate, and acoustic signal from AE sensor. Those were captured and recorded in time domain.

With the proposed method, the rest period will be recognized as a priority. According to a determination of the rest period required when no bubble remained in electrolytic solution, equations (1) and (2) were used with some calculation details: 10A activation current, 200 micron bubble diameter, 5 centimetres of averaged distance when bubble travels to the top of electrolyte surface, and the gas bubble set as oxygen (higher density than hydrogen; rising time would be dominant). It is 
found that at least 455 milliseconds of rest period is needed to meet the requirement. Nevertheless, the actual rest period measured with the AE sensor was about 150 millisecond (red border in Fig. 2), the difference in the results depends on the bubble size in the major bubbles.
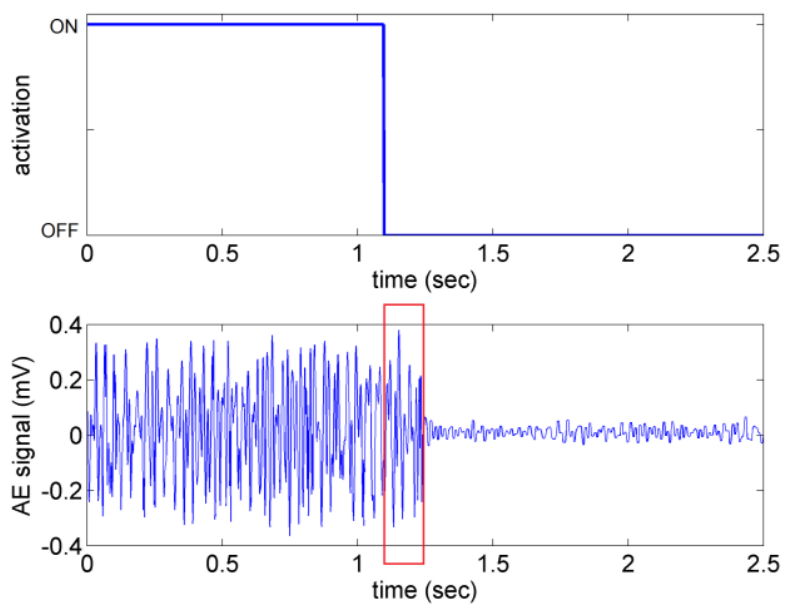

Figure 2. Time-domain acoustic emission of bubble breaking

Having considered on the bubble size using the acoustic signal measurement during bubble rising on the rest period, the frequency response was shown in Fig. 3. There are two controversial frequency bandwidths at 120 $\mathrm{kHz}$ and $260 \mathrm{kHz}$. By the equation (3), the two represented frequencies figure that the bubbles consist of two major bubble sizes. Oxygen bubbles reflect lower bandwidth and hydrogen bubbles attribute higher frequency.

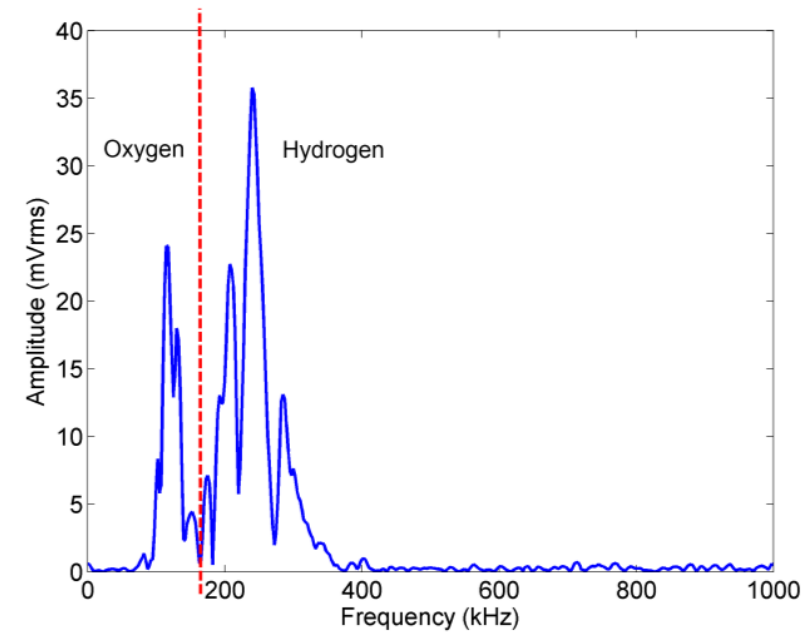

Figure 3. Acoustic frequency responses of bubble breaking.

\subsection{Production efficiency}

From the experimental results, only hydrogen production efficiency was considered to determine heating power as the output with the electrical power input. As shown in Fig. 4, the production efficiency presented in terms of rest period varying from 2 milliseconds to 800 milliseconds with the activations of $10,20,30,40$, and $50 \mathrm{~A}$ peak current and the frequencies of $1,5,10,50$, and $100 \mathrm{~Hz}$. Due to the rest period and the activation frequency has algebraic relationship and the large variation of rest period, the $\mathrm{x}$ axis also represent as logarithmic scale. According to rest period variation, the results can be grouped by activation frequency ranges and shown as almost linear relationship to the production efficiency. In the other words, each of activation frequency has individual of proper rest period. Focusing on the activation current $30 \mathrm{~A}_{\text {peak }}$ at the 160 millisecond rest period, this point was the highest efficiency and interesting that the rest period was approximate to the rest period requirement obtained from $\mathrm{AE}$ measurement. However, the variation of rest period cannot simply clarify in terms of the efficiency explanation, so the rest period was represented by duty cycle (rational of activation period and cyclic period) for easy interpretation and practical setup in the future.

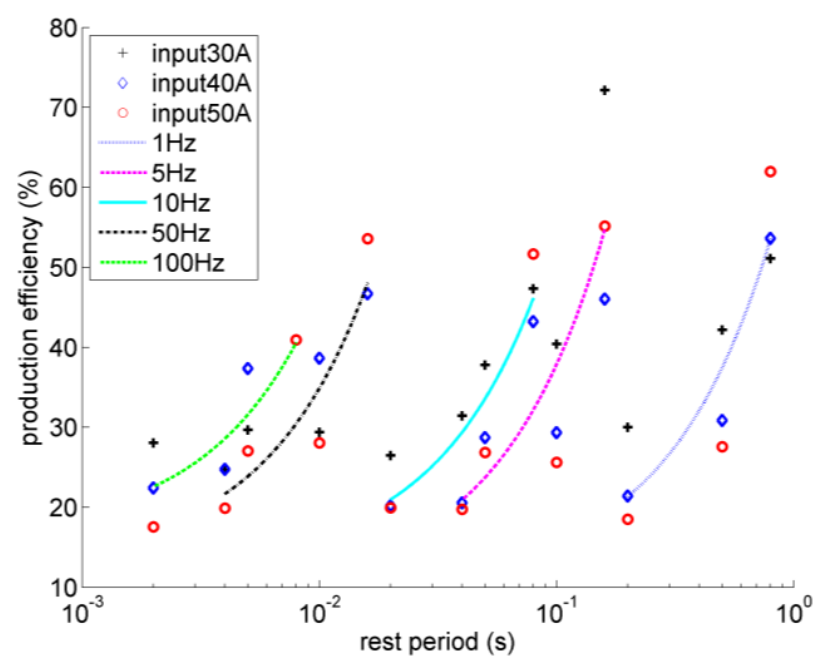

Figure 4. Production efficiency in terms of rest period in various frequencies.

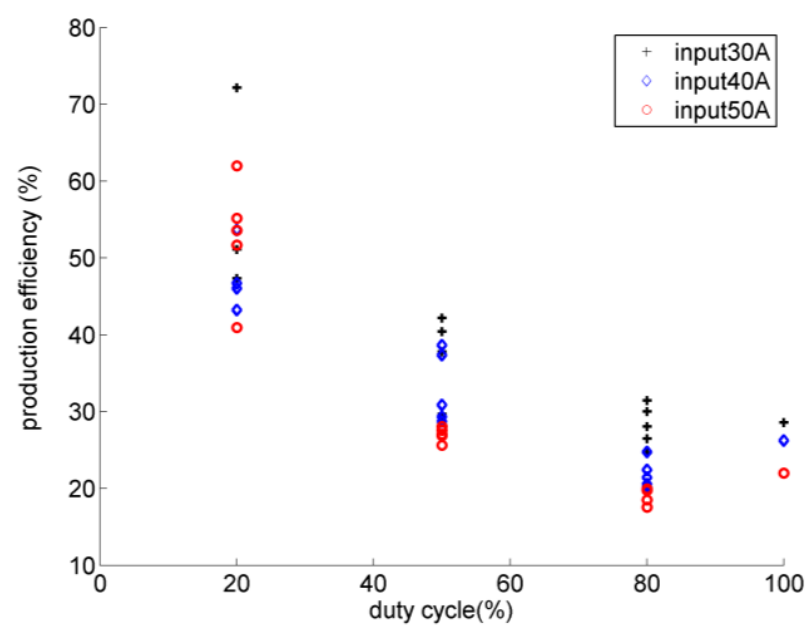

Figure 5. Production efficiency in term of duty cycle

The relationship between production efficiency and duty cycle was shown on Fig. 5. At the same result of rest period variation, duty cycle was represented instead. The pulse-activated was set on $20 \%, 50 \%, 80 \%$, and $100 \%$ duty cycle, with frequency $1,5,10,50$, and $100 \mathrm{~Hz}$. The markers represent as peak current amplitude scattered on production efficiency and duty cycle plane. Similar to Fig. 
4, duty cycle cannot describe the optimum operating condition of the pulse activated, but the activation with lower duty cycle tends to higher efficiency especially in lower activation peak current. Moreover, some of pulseactivated lack of efficiency improvement ability than the $100 \%$ duty cycle (continuous activation), that meant this is not always a suitable choice for improving efficiency of electrolysis hydrogen production. However, the operation with lower duty cycle must be concerned.

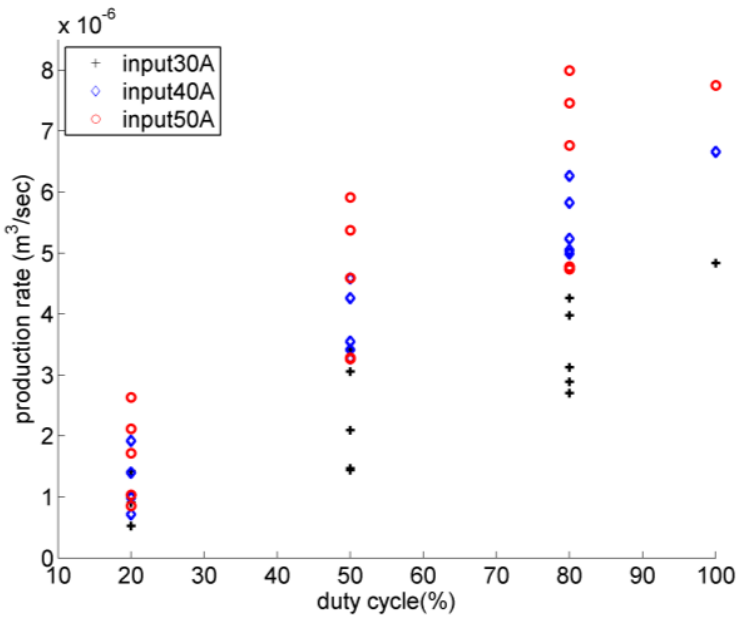

Figure 6. Hydrogen production rate in terms of duty cycle

\subsection{Production rate and resistivity}

Taking into account on the hydrogen production rate, it is known that the bubbles created reduce the active area of the electrodes and increase resistivity of the system, the result also presented in difference to the efficiency. Fig. 6 presents the relationship of hydrogen production rate as a function of activation duty cycle, by the increase of duty cycle, the production rate also has a tendency to increase according to Faraday's law of electrolysis. The high activation current amplitude and/or high duty cycle gives high production rate which is proportional to the averaged input current. In Fig. 6 there are some scatters of production rate at the same current and same duty cycle values. This is because of the effect of activation frequency. The lower frequency tends to achieve higher production rate. In comparison with continuous activation, the production rate of a low frequency pulse-activated was preferable because the lower frequency has longer rest period and makes bubble coverage clear from the electrodes to get ready for activation. The advantage on production rate of the lower frequency activation scheme was consistent with the use of low frequency on the view of efficiency. Corresponding to Fig. 5, the low frequency provides higher efficiency at the same duty cycle. Our conclusion is that the selected activation regime as low frequency and low duty cycle has been proved to be suitable for the electrolyzer setup.

In the previous study, increasing of activation current resulted in more fine bubble [12]. Agree with the considering on resistivity and bubble size relationship [13], too small bubble size trend to higher resistivity but the big bubble was consistent. Therefore, the increase of activation current was relative to higher resistivity due to bubble size effect. Fig. 7 demonstrates the resistivity of electrolytic solution during pulse-activated rest period as a function of average input current. The small increase rate of resistivity meant that there is a little change of bubble size. In accordance of AE bubble's bandwidth measuring on the experiment, the breaking frequency described that there was a small variation of bubble size due to the activation current increase. Hence, the smaller bubble due to increase of activation current got slower rising velocity and the resistivity also increase. Likewise the result in efficiency, the low activation frequency gives better advantage.

Consequently, the use of pulse-activated in industrial application should consider both production rate and efficiency. The use of pulse-activated for efficiency enhancement but remain production rate as continuous activation needs to activate multiple cells in sequence. However, in the industrial application hydrogen production efficiency was not only determine as production efficiency, but the efficiency in the rational of installation space was necessary.

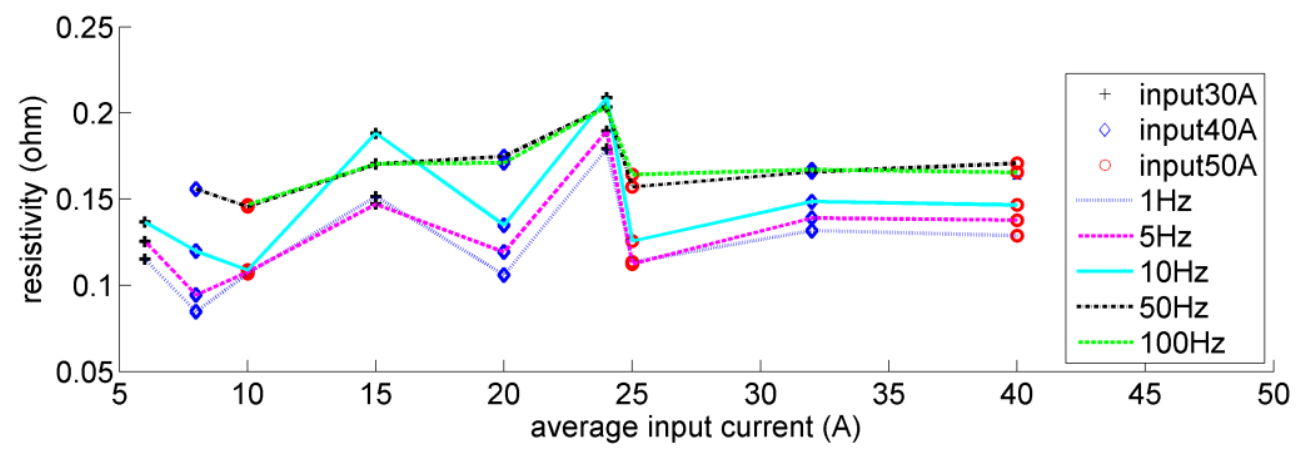

Figure 7. Resistivity of electrolytic solution during pulse-activated rest period in terms of average input current

Though, the pulse activated electrolysis hydrogen production takes more installation space than the conventional, but it gains higher efficiency and gives electrode's life time longer due to less crystal deposited on electrode surfaces [6].

\section{Conclusion}

The experimental results confirm the strong effect of bubble interacting with production efficiency. The lower frequency is advantageous in long rest period according 
to bubble rising time while the longer rest period makes productivity depreciated. So, the minimum rest period for free rising bubble, until empty, needs to be determined. In the study, the activation scheme comprising $30 \mathrm{~A}$ activation current at $5 \mathrm{~Hz}$ activation frequency and the rest period of 160 millisecond gives the best result and agreed with the least rest period that achieved from AE records. Duty cycle has been proposed to use as a practical setup parameter for simple analysis. Similar to the result emerging on the rest period, the production efficiency corrupts when increasing duty cycle. According to the rest period of 160 millisecond, the same activation scheme of pulse-activated shows higher efficiency than others, in contrast, simultaneously the other lower activation currents have a tendency to gain higher efficiency.

Moreover, the lower frequency tends to achieve higher production rate because the pulse-activated help the bubbles detaching from the electrode easier and increase active area. The experiment was given the effective criteria to use pulse-activated on electrolysis hydrogen production by controlling activation scheme with low frequency and low duty cycle. With the considering on the resistivity, the higher resistivity caused higher in Ohmic's loss and efficiency reduction. The use of lower activation frequency affected the lower resistivity similar to the production efficiency.

According to Faraday's law of electrolysis, the gas production is proportional to the averaged input current. So, the increase of duty cycle leads the production rate has a tendency to increase and vice versa. Definitely, the volumetric gaseous produced by electrolysis was strictly conformed to the Faraday's law, so the bubble size was the dominant parameter that indicated remaining voidage within specific time.

To manifest the bubble interact with electrolysis reaction, the overpotential regarding to bubble size requires a further study for the most effective activation procedure on bubble size controlling.

\section{Acknowledgement}

This materials based on acoustic emission testing is supported by Maintenance Technology Center (MTC), King Mongkut's University of Technology Thonburi.

\section{References}

1. K. Mazloomi, N.B. Sulaiman, and H. Moayedi, Int. J. Electrochem. Sci., 7, 3314-3326, (2012).

2. K. Mazloomi, N.B. Sulaiman, and H. Moayedi, Int. J. Electrochem. Sci., 7, 3466-3481, (2012).

3. B. Mandal, A. Sirkar, A. Shau, et al., Int. J. Renew. Energy Res., 2, 99-102, (2012).

4. K. Zeng, and D. Zhang, Prog Energy Combust Sci., 36, 307-326, (2010).

5. L.J.J. Jansseen, and E. Barendrecht, Electrochem. Acta, 28, 341-346, (1983).

6. D. Hristova, I. Betova, and T. Tzvetkoff, EMCR2012, (2012).

7. A.A. Kendoush, Chem. Eng. Sci., 56, 235-238, (2001).

8. N. Shimizu, S. Hotta, T. Sekiya, et al., J. Appl. Electrochem., 36, 419-423, (2005).

9. N. Kircheva, S. Tant, B. Legros, S. Genies, et al., 30th EWGAE and 7th ICAE, (2012).

10. K. Wu, S. Lee, W. Lee, et. al., TMAM 2015, 33-37, (2015).

11. C. Jirarungsatian, A. Prateepasen, Corros. Sci., 52, 187-197, (2010).

12. Khosla, N.K., and Venkatachalam, S., Pulsed Electrogeneration of Bubbles for Electroflotation, Journal of Applied Electrochemistry, vol. 21, pp. 986-990, (1991).

13. K. Tangphant, K. Sudaprasert, S. Channarong, Russ J Electrochem, 50, No. 3, 253-259, (2014). 